\title{
FDI, Licensing, e Crescimento da Produtividade Total de Fatores*
}

\author{
Ana Flavia Bonzanini ${ }^{\dagger}$, Eduardo Correia de Souza ${ }^{\ddagger}$, Leonardo Melo ${ }^{\S}$
}

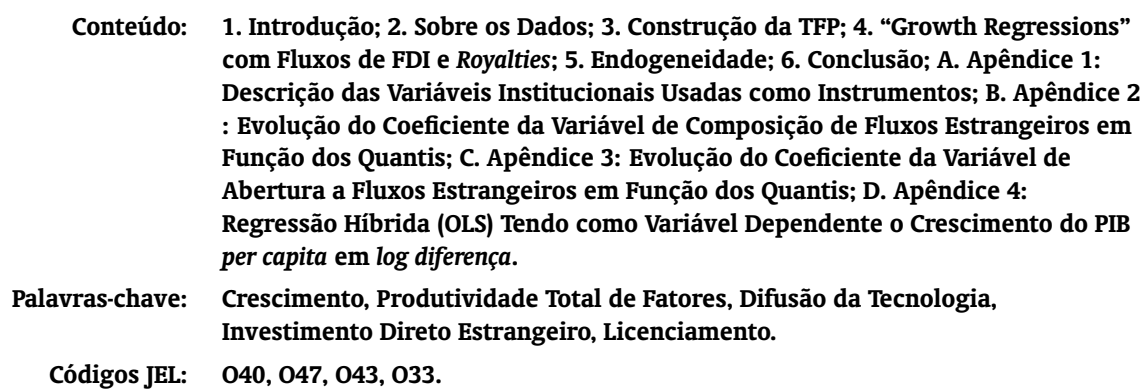

Neste trabalho analisamos os impactos de dois canais de transferência internacional de tecnologia, investimento direto estrangeiro e licenciamento (FDI e licensing), sobre o crescimento da produtividade total de fatores (TFP) numa amostra de 88 países, para o período 1980-2006. Em relação a Pessoa (2008), trazemos aqui três contribuições originais: incluímos na especificação do modelo econométrico uma variável de composição dos fluxos estrangeiros de capital; nossa amostra compreende também países pobres e em desenvolvimento, e não só da $\mathrm{OCDE}$; oferecemos um tratamento do problema de endogeneidade típico em regressões de crescimento tendo como variáveis explicativas medidas do tipo "abertura financeira" dos países.

We analyze the impacts of two international technology transfer channels, foreign direct investment (FDI) and licensing, on total factor productivity (TFP) growth in a sample of 88 countries for the period 1980-2006. Comparing with Pessoa (2008), we bring here three original contributions: we include in the econometric model specification a variable which captures the composition of foreign capital flows; our sample comprises poor and developing countries too, not only OECD's; we offer a

\footnotetext{
*Gostaríamos de agradecer a Fábio Augusto Reis Gomes e a um parecerista anônimo pelos comentários.

$\dagger$ Insper - Instituto de Ensino e Pesquisa.

‡Professor do Insper - Instituto de Ensino e Pesquisa. Endereço para correspondência: Rua Quatá, 300, Vila Olímpia. CEP 04546-042. São Paulo - SP. E-mail: eduardocs@insper . org . br

$\S$ Insper - Instituto de Ensino e Pesquisa.
} 
treatment for the typical endogeneity problem found in growth regressions which have as explanatory variables measures like countries' "financial openness".

\section{INTRODUÇÃO}

Como se sabe, a difusão internacional de tecnologia é um importante condicionante do processo de convergência/divergência de renda per capita entre os países. No limite, sob uma difusão perfeita da tecnologia, todos os países possuiriam a mesma produtividade total de fatores (TFP), e então diferenças de renda per capita seriam explicadas apenas por diferenças nas dotações de fatores, como capital humano e capital físico. Dentre os diversos mecanismos pelos quais se dá a difusão internacional de tecnologia como, por exemplo, o livre fluxo de bens e idéias entre os países, dois deles se destacam por parecerem claramente substitutos, pelo menos a nível microeconômico: investimento direto estrangeiro (foreign direct investment ou FDI, doravante) e licensing.

Considere uma firma inovadora localizada num país desenvolvido que tem interesse em transferir a manufatura de seu novo produto para um país menos desenvolvido, porque ali os custos da mão-de-obra são menores. Essa firma pode ou bem abrir uma filial no país menos desenvolvido, auferindo com isso dividendos na forma de remessas de lucro (FDI); ou bem autorizar através de um contrato outra firma, localizada no país menos desenvolvido, a produzir o novo bem em troca de pagamentos de royalties (licensing). A escolha dentre esses dois mecanismos é modelada explicitamente em Glass e Saggi (2002) e em Antràs (2005), e basicamente depende de aspectos como a qualidade dos contratos e dos sistemas jurídicos dos países menos desenvolvidos (que afeta a divisão dos lucros ou rendas entre licenciadores e licenciados), direitos de propriedade intelectual (que afetam a probabilidade de imitação por firmas rivais no país receptor da tecnologia), o grau de "estandardização" do novo produto, etc.

Apesar desses avanços na teoria sobre FDI versus licensing, poucos são os trabalhos empíricos que relacionam ambos os mecanismos com o crescimento econômico ou progresso tecnológico dos países. Por exemplo, Woo (2009) é um dos primeiros a encontrar uma relação positiva entre fluxos de FDI e crescimento da TFP, numa amostra de 92 países para o período de 1970-2000. Embora esse trabalho traga uma contribuição significativa para a literatura ao usar como variável dependente a TFP e não a renda per capita (o que equivale a controlar para o efeito do FDI sobre a renda via aumento do estoque de capital), Woo (2009) não inclui na sua análise os fluxos de royalties e taxas de licenciamento. Essa lacuna na literatura é preenchida por Pessoa (2008): utilizando um painel de 16 países da OCDE no período de 1985 a 2002, o autor encontra um impacto positivo tanto do FDI recebido quanto dos royalties pagos pelos países sobre as suas TFPs. Além disso, ao incluir no seu modelo econométrico um termo de interação entre FDI e royalties (licensing), Pessoa (2008) encontra uma relação de substituição a nível macro entre esses dois mecanismos de difusão internacional de tecnologia.

Um resultado de Pessoa (2008) que nos interessa particularmente é que em algumas das especificações de seu modelo econométrico encontra-se uma elasticidade maior (do nível) da TFP em relação aos royalties e license fees do que em relação ao FDI. Isso nos sugere uma questão que pode ser colocada da seguinte maneira: que países tiveram maior crescimento da TFP, os que se basearam mais em FDI ou os que se basearam mais em licensing ao longo do processo de industrialização/crescimento? ${ }^{1}$

\footnotetext{
${ }^{1}$ Ao nível das evidências anedóticas, essa questão encontra uma resposta no trabalho de Cyhn (2002). Apoiando-se em diversos estudos de caso de empresas de alta tecnologia, Cyhn propõe que o sucesso do desenvolvimento da Coréia (do Sul, evidentemente) não foi fruto de FDI, mas em grande parte derivado do processo de OEM (Original Equipment Manufacturing), que pode ser definido como uma forma de subcontratação internacional onde a firma manufatureira (do país receptor de tecnologia) produz bens finais para a firma estrangeira que venderá os mesmos sob a sua marca. Tal forma de transmissão de conhecimento teria, segundo o autor, maiores spillovers tecnológicos devido à possibilidade de que a firma manufatureira entre em contato com a tecnologia sob a supervisão da firma detentora desta.
} 
É nesse ponto que se insere o nosso trabalho: como testar empiricamente a conjectura de que a transferência de tecnologia via licensing é mais (ou menos) favorável do que a via FDI ao crescimento da TFP de um país receptor? Basicamente, vamos usar aqui a mesma abordagem de Aghion et alii (2005) ao analisarem o impacto do "desenvolvimento financeiro" dos países sobre o crescimento da renda per capita. Esses autores incluem, além da tradicional variável “crédito (total) sobre o PIB", uma variável chamada de "composição do crédito" (razão crédito privado sobre crédito público) do lado direito da equação de crescimento, e encontram que as duas variáveis têm coeficientes significativos e positivos. Analogamente, aqui vamos usar como "variáveis do lado direito" uma medida de abertura de um país para fluxos estrangeiros que será a soma de FDI recebido e royalties pagos dividida pelo PIB do país, e também uma medida da composição desses fluxos, dada pela razão royalties sobre FDI.

Além dessa diferença de especificação do modelo econométrico (com ênfase no crescimento ao invés de no nível da TFP, e adotando a composição royalties/FDI como uma variável explicativa), trazemos aqui duas contribuições adicionais em relação a Pessoa (2008):

- nossa amostra é mais abrangente, com 88 países, para o período 1980 a 2006. A inclusão de países em desenvolvimento, e não só da OCDE, parece-nos particularmente relevante no contexto da difusão internacional de tecnologia.

- lidamos com um problema de endogeneidade que provavelmente acomete todas as growth regressions que têm no lado direito variáveis como "abertura comercial", "desenvolvimento financeiro", "abertura para fluxos de FDI", etc.: no nosso contexto, um país teve maior crescimento porque foi mais aberto para FDI, ou vice-versa? Aqui, procuramos resolver esse problema usando variáveis instrumentais (2SLS): intuitivamente, esperamos que medidas de impostos sobre remessas de lucros e de risco de expropriação/estatização sejam relevantes para explicar a abertura para fluxos estrangeiros, em particular o volume de FDI. Por outro lado, dados relativos à qualidade dos contratos, assim como alguma outra medida de simetria de tratamento jurídico entre firmas domésticas e estrangeiras seriam bons instrumentos para explicar a composição dos fluxos de investimentos, em particular o volume de licensing. Diante da escassez de dados sobre essas "variáveis institucionais", acabamos por utilizar como instrumentos os índices encontrados nos trabalhos de Ginarte e Park (1997), Zhao (2006) e Park (2008), além do relatório Doing Business do Banco Mundial.

O restante deste artigo está organizado da seguinte maneira: a Seção 2 apresenta os dados que serão utilizados; a Seção 3 descreve a metodologia de construção da série histórica de TFP, a partir do trabalho de Ferreira et alii (2010); a Seção 4 apresenta os resultados da estimação do modelo básico de regressão da taxa de crescimento da TFP contra as variáveis "abertura" e "composição" acima descritas, numa cross-section de 80 países; a Seção 5 apresenta o tratamento do problema de endogenidade com variáveis instrumentais; a Seção 6 conclui.

\section{SOBRE OS DADOS}

No que diz respeito às medidas de FDI e de royalties normalmente adotadas na literatura, vale notar que existe certa inconsistência entre elas, pois o FDI é medido através de fluxos de entrada de capitais para o país onde se está investindo, ao passo que o licensing é medido pelo pagamento de royalties que são um fluxo de saída de capitais do país que recebeu a tecnologia. Ou seja, ao medir somente o fluxo de entrada de FDI, trabalhos como o de Pessoa (2008) estariam captando o elemento esporádico do FDI, que é o investimento inicial, e comparando-o com o elemento sistemático do licensing que é o pagamento de royalties. Nossa ideia original seria então que neste trabalho se utilizasse a remessa de lucros de firmas estrangeiras instaladas no país como proxy para a quantidade de FDI, proxy que seria diretamente comparável aos pagamentos de royalties. No entanto, como tal dado não está disponível para um número suficientemente grande de países, utilizou-se a medida de FDI flows fornecida pela

RBE Rio de Janeiro v. 67 n. 1 / p. 25-43 Jan-Mar 2013 
UNCTAD. Tal medida capta o valor total, em dólares correntes, que um país recebeu em investimento direto estrangeiro.

Os dados relativos aos royalties foram também encontrados na base da UNCTAD. No entanto, tal base é pouco extensa no tempo, com as informações se iniciando no ano de 1980, que será, portanto, o ano inicial das nossas regressões. Finalmente, como a nossa variável que capta a abertura da economia é uma proporção do PIB, o PIB em valores correntes também é proveniente da base de dados da UNCTAD.

A partir dos dados anuais relativos aos FDI flows e aos royalties, foram construídas as variáveis $A_{i} \equiv\left(\frac{\text { royalties }+F D I}{P I B}\right)_{i}$ e $c p_{i} \equiv\left(\frac{\text { royalties }}{F D I}\right)_{i}$ para cada país $i$; estas variáveis correspondem respectivamente à média do grau de abertura e à média da composição dos fluxos entre 1980 e $2006{ }^{2}$

É importante destacar que, dos 141 países da base original da UNCTAD, para vários deles as informações relativas aos royalties são inexistentes entre 1980 e 1990. Nesse caso específico, optou-se por retirar esses países da amostra. Também removemos da amostra alguns países outliers que se destacam por terem uma proporção de royalties sobre FDI flows acima ou próximo de $200 \%$, sendo que a média da amostra é de $35 \%$ e o erro padrão de $15 \%$.

Tabela 1: Análise de dados - Comparação entre as proporções de royalties sobre FDI flows (variável $c p$ ) com e sem outliers

\begin{tabular}{lcc}
\hline & Composição & Abertura \\
\hline Média & $35 \%$ & $17 \%$ \\
Erro padrão & $15 \%$ & $3 \%$ \\
Mediana & $7 \%$ & $7 \%$ \\
Desvio padrão & $167 \%$ & $29 \%$ \\
Curtose & $11283 \%$ & $1089 \%$ \\
Assimetria & $1039 \%$ & $315 \%$ \\
Mínimo & $-7 \%$ & $-7 \%$ \\
Máximo & $1838 \%$ & $168 \%$ \\
\hline
\end{tabular}

Fonte: UNCTAD, Penn World Table 7.0.

Como já discutimos na Introdução, a variável dependente das nossas regressões é a taxa de crescimento da TFP dos países. E uma vez que a construção da série de TFP requer uma metodologia própria que será detalhada na próxima seção, limitamo-nos aqui a listar os dados que foram utilizados nessa construção.

Em primeiro lugar, como medida de "capital humano" utilizaremos os dados desenvolvidos por Barro e Lee (2001), especificamente o número médio de anos de estudo para a população economicamente ativa acima de 15 anos de idade. ${ }^{3}$ Assim como os dados sobre FDI e royalties, esses dados educacionais estão disponíveis para um número relativamente restrito de países, fazendo com que ao cabo o número de observações da regressão cross-country deste estudo fosse de 88 países; número que ainda assim é expressivo uma vez que Aghion et alii (2005) utilizam dados de 76 países no seu estudo.

Para o produto per capita, a taxa média de poupança em capital físico e a taxa de crescimento populacional, utilizamos respectivamente as variáveis denominadas "rgdpch", "ki" e "POP" da Penn World Table 7.0.

\footnotetext{
${ }^{2}$ Os dados completos referentes às variáveis $A$ e $c$ p, para cada país da nossa amostra, podem ser obtidos a pedido do leitor.

${ }^{3}$ Como essa base de dados fornece informações do ano de 1960 a 2000, num intervalo de cinco anos, interpolamos os dados para criar uma série contínua.
} 
O valor escolhido para a taxa de crescimento da fronteira tecnológica $(g)$ foi $2 \%$ ao ano, como em Mankiw et alii (1992); e para a taxa de depreciação do capital físico $(\delta)$ foi $7 \%$ ao ano, como em Ferreira et alii (2010).

Finalmente, a fim de tratarmos do problema de endogeneidade envolvendo as variáveis crescimento da TFP, "abertura" $(A)$ e "composição" $(c p)$, foi preciso escolher como instrumentos algumas "variáveis institucionais" que nos parecessem adequadas. Três estudos foram decisivos para nortear a escolha de tais variáveis: O primeiro deles foi o de Zhao (2006), que para capturar o ambiente político e legal dos países utiliza índices como o The Law and Order Index e o Property Protection Index; e para capturar os direitos de propriedade intelectual dos países utiliza o GP Index. Os outros dois estudos foram: o conduzido por Ginarte e Park (1997), e Park (2008). ${ }^{4}$ Com isso, foram encontrados oito instrumentos: 
Tabela 2: Análise de dados - Variáveis institucionais

\begin{tabular}{lccc}
\hline & GP Index & $\begin{array}{c}\text { Investment } \\
\text { Freedom }\end{array}$ & $\begin{array}{c}\text { Strength of } \\
\text { Investor's }\end{array}$ \\
\hline Média & 3,611 & 56,750 & 5,430 \\
Erro padrão & 0,087 & 2,073 & 0,177 \\
Mediana & 3,680 & 50,000 & 5,300 \\
Desvio padrão & 0,779 & 18,540 & 1,582 \\
Curtose & 2,263 & 2,521 & 3,254 \\
Assimetria & $-0,357$ & 0,051 & 0,622 \\
Mínimo & 1,780 & 10,000 & 2,300 \\
Máximo & 4,880 & 90,000 & 9,700 \\
\hline Fontes: Doing Business, Ginarte e Park (1997) e Park (2008), ICRG Risk \\
Rating System.
\end{tabular}

\section{CONSTRUÇÃO DA TFP}

A função de produção é

$$
y_{i}=A_{i} k_{i}^{\alpha} H_{i}^{1-\alpha}
$$

onde $y_{i}$ é a renda per capita do país $i$; $A$ é a produtividade total dos fatores (TFP); $k$ o estoque de capital por trabalhador; $H$ é o estoque de capital humano; e $\alpha$ é a participação do capital na renda. Assumimos que $\alpha=0,4$, como em Ferreira et alii (2010).

A primeira etapa para a construção da série é a determinação de $H$. Seguindo Bills e Klenow (2000), assume-se que:

$$
H_{i t}=e^{\phi\left(h_{i t}\right)}
$$

onde $h_{i t}$ é o número médio de anos de estudo da população economicamente ativa do país $i$ e $\phi$ é um parâmetro tal que:

$$
\phi(h)=\frac{\theta}{1-\psi} h^{1-\psi}
$$

$\operatorname{com} \theta=0,32$ e $\psi=0,58$.

O segundo passo é a determinação de $k$, estoque de capital por trabalhador. Aqui empregamos o método do inventário perpétuo, com

$$
K_{t}=(1-\partial) K_{t-1}+I_{t-1}^{8}
$$

com $K_{t}$ como estoque agregado de capital e $I_{t}$ como investimento agregado no período $t . \partial=7 \%$ é a taxa de depreciação do capital. A fim de obter um valor para o estoque de capital inicial, $K_{0}$, adotamos a usual "hipótese BEA" (Bureau of Economic Analysis): em steady-state o investimento agregado estaria

\footnotetext{
${ }^{8}$ É imediato transformar a expressão (4) para expressá-la em termos do capital por trabalhador: $k_{t}=$ $\frac{L_{t-1}}{L_{t}}\left[(1-\partial) \frac{K_{t-1}}{L_{t-1}}+\frac{I_{t-1}}{L_{t-1}}\right]$.
} 
crescendo a uma taxa $g+n$, com $g$ sendo a taxa de crescimento da fronteira tecnológica e $n$ a taxa de crescimento populacional, de modo que

$$
I_{t}=I_{0} \cdot[(1+g) \cdot(1+n)]^{t}
$$

Iterando (4) para trás e usando (5), é simples mostrar que o estoque de capital inicial é dado por:

$$
K_{0}=\frac{I_{0}}{(g+n+n g+\partial)}
$$

onde $I_{0}$ é o investimento total inicial e $n$ o crescimento populacional (que será diferente para cada país, de modo que a notação completa seria $n_{i}$ ). Assim como em Ferreira et alii (2010), considera-se que o investimento agregado inicial é uma média dos investimentos totais dos cinco primeiros anos da série:

$$
\frac{I_{0}}{L_{0}}=\frac{1}{5}\left(\frac{I_{0}}{L_{0}}+\frac{I_{1}}{L_{1}(1+g)}+\frac{I_{2}}{L_{2}(1+g)^{2}}+\frac{I_{3}}{L_{3}(1+g)^{3}}+\frac{I_{4}}{L_{4}(1+g)^{4}}\right)
$$

Para chegarmos a $L$ (número de trabalhadores), utilizamos as variáveis rgdpch (Real GDP Chain per capita) e rgdpwok (Real GDP Chain per worker) da Penn World Table 7, de modo que $L$ é o produto da taxa de participação $(p)$ pela população:

$$
\begin{gathered}
L=p \cdot P O P \\
p=\frac{r g d p c h}{r g d p w o k}
\end{gathered}
$$

Assim, uma vez construídas as séries relativas aos diferentes fatores da função de produção em (1), bastou rearranjá-los de forma a obter a série da TFP:

$$
A_{i t}=\frac{y_{i t}}{k_{i t}^{\alpha} H_{i t}^{(1-\alpha)}}
$$

\section{4. “GROWTH REGRESSIONS” COM FLUXOS DE FDI E ROYALTIES}

\subsection{Especificação do modelo}

O ponto de partida ou modelo básico de growth regression que vamos utilizar encontra-se em Aghion et alii (2005):

$$
g_{y i}(0, t)=\beta_{0}+\beta_{1} \cdot \ln y_{i 0}+\beta_{2} \cdot \ln s_{k i}+\beta_{3} \cdot \ln s_{H i}+\beta_{4} \cdot \ln \left(n_{i}+g+\delta\right)+\beta_{5} \cdot F_{i}+\beta_{6} \cdot c c_{i}+\varepsilon_{i}
$$

onde

$g_{y i}(0, t)$ é a taxa média anual de crescimento da renda per capita do país $i$ no intervalo de tempo $[0, t]$, por exemplo de 1980 a 2006;

$y_{i 0}$ é a renda per capita inicial do país $i$;

$s_{k i}$ é a taxa média (no intervalo de 0 a $t$ ) de investimento em capital físico do país $i$;

$s_{H i}$ é a taxa média de investimento em capital humano de $i$;

$n_{i}$ é a taxa média de crescimento populacional de $i$; 
$g$ é a taxa de progresso tecnológico da fronteira, $2 \%$ a.a.;

$\partial$ é a taxa de depreciação do capital físico;

$F_{i}$ é a proxy para “desenvolvimento financeiro”, usualmente crédito sobre PIB;

$c c_{i}$ é a razão crédito privado sobre crédito público.

Neste trabalho, analogamente, usaremos as seguintes duas variáveis do lado direito: a primeira, $A_{i} \equiv((\text { royalties }+F D I) / P I B)_{i}$ é a nossa medida de abertura da economia para fluxos estrangeiros para um determinado país $i$, correspondendo a $F$ em (11); a segunda, $c p_{i} \equiv(\text { royalties } / F D I)_{i}$ é a nossa medida de composição dos fluxos, correspondendo a $c c$ em (11). Além disso, como estamos interessados nos efeitos do licensing e do FDI sobre o nível tecnológico dos países, e não sobre a formação do estoque de capital, nossa variável dependente será a taxa média de crescimento da TFP e não a da renda per capita. Dada a metodologia de construção da TFP descrita na Seção 3, devemos alterar o lado direito da regressão, retirando os controles $s_{k i}, s_{H i}$ e $n_{i}$. Com o que chegamos simplesmente a

$$
g_{T F P_{i}}(0, t)=\beta_{0}+\beta_{1} \cdot A_{i}+\beta_{2} \cdot c p_{i}+\varepsilon_{i}
$$

onde a taxa de crescimento da TFP é calculada em log difference: $g_{T F P_{i}}(0, t)=$ $\left(\ln T F P_{i t}-\ln T F P_{i 0}\right) /(t-0)$.

Assim, nossa pergunta inicial "que países tiveram maior crescimento da TFP, os que se basearam mais em FDI ou os que se basearam mais em licensing ao longo do processo de industrialização/crescimento?" é respondida testando o sinal de $\beta_{2}$. A partir do modelo básico em (12), procuramos também aprimorar a especificação da regressão baseando-nos na literatura sobre convergência. Miller e Upadhyay (2002) encontram evidências quanto à significância da variável "TFP inicial”. Desse modo, uma melhor especificação seria:

$$
g_{T F P_{i}}(0, t)=\beta_{0}+\beta_{1} \cdot A_{i}+\beta_{2} \cdot c p_{i}+\beta_{3} \cdot \ln T F P_{0 i}+\varepsilon_{i}
$$

onde $T F P_{0 i}$ representa a TFP do país $i$ no ano inicial. A hipótese de convergência é $\beta_{3}<0$.

Finalmente, artigos seminais do crescimento endógeno como Grossman e Helpman (1991) destacam que países com um maior nível de capital humano teriam maiores possibilidades de se engajar em atividades de pesquisa e de absorver o conhecimento necessário para fazer uso das inovações tecnológicas. Woo (2009), que procura colocar em evidência os impactos do FDI sobre a TFP, propõe um modelo de regressão cuja variável dependente é a TFP e onde são incluídas no lado direito variáveis como a TFP inicial e o estoque de capital humano. A exemplo desse autor, estimaremos também:

$$
g_{T F P_{i}}(0, t)=\beta_{0}+\beta_{1} \cdot A_{i}+\beta_{2} \cdot c p_{i}+\beta_{3} \cdot \ln T F P_{0 i}+\beta_{4} \cdot \ln s_{H i}+\varepsilon_{i}
$$

\subsection{Resultados empíricos}

Ao estimarmos os modelos em (12), (13) e (14), estamos lidando com regressões cross-country, onde tipicamente a heterocedasticidade dos resíduos tende a ser mais significativa, fato confirmado para as especificações que correspondem às expressões (13) e (14), fazendo-nos adotar, nestes dois casos, estimadores de White. Seguem os resultados para a nossa amostra mais reduzida (compatível com as regressões 2SLS que faremos a seguir), de 80 países:

Observa-se que a variável $c p$ ("composição", na Tabela 3) se mostra significante a 5\% em duas regressões e significante a $1 \%$ na especificação correspondente a (13). O sinal do coeficiente associado a $c p$ é sempre positivo. Esses resultados confirmam nossa conjectura de que países que se apoiaram mais em licensing do que em FDI tiveram maior crescimento da TFP. Apesar disso, o impacto da outra variável, A ("abertura", na Tabela 3) é quase dezessete vezes maior se considerada a especificação em (14). 
Tabela 3: OLS - Regressões tendo como variável dependente a taxa média anual de crescimento da TFP em log diferença

\begin{tabular}{cccc}
\hline \multicolumn{4}{c}{ Taxa de crescimento da TFP (1980 a 2006), amostra com 80 países } \\
\hline Especificação usada & Modelo (12) & Modelo (13) & Modelo (14) \\
\hline \multirow{2}{*}{ Composição } & $0.007284^{* *}$ & $0.008715^{*}$ & $0.005823^{* *}$ \\
& $(0.007284)$ & $(0.002171)$ & $(0.002262)$ \\
Abertura & $0.126937^{*}$ & $0.123820^{*}$ & $0.097399^{*}$ \\
& $(0.036976)$ & $(0.031564)$ & $(0.032223)$ \\
ln TFP inicial & & $-0.008289^{* *}$ & $-0.009774^{* *}$ \\
& & $(0.004141)$ & $(0.004035)$ \\
ln Sh & & & $0.005643^{* *}$ \\
& & & $(0.002154)$ \\
Constante & $\left(0.004158^{* *}\right.$ & $0.033979^{* * *}$ & $0.054877^{*}$ \\
& & $(0.019081)$ & $(0.200078)$ \\
\hline
\end{tabular}

Nota: erros-padrão em parênteses.

*, ** $\mathrm{e}^{* * *}$ para significante a $1 \%, 5 \%$ e $10 \%$ respectivamente.

Fontes: Unctad, Barro e Lee (2001) e Penn World Table 7.0.

\subsection{Robustez}

Segundo Hallock e Koenker (2001), ao se realizar uma regressão quantílica pode-se verificar se o intervalo de confiança da mediana contém a estimativa por OLS: se essa estimativa estiver contida no intervalo de confiança, deduz-se que ela não é distorcida pela presença de outliers na série de dados. Assim, rodamos regressões quantílicas para a nossa especificação mais completa (14). Para a variável "composição" $(c p)$, não houve diferença significativa entre os coeficientes obtidos por OLS e o coeficiente da mediana obtido pela quantílica (ver Apêndice 2). Ademais, ao se considerar o intervalo de confiança não há diferença entre os coeficientes em nenhum quantil.

Já ao se realizarem os testes sobre a variável "abertura" $(A)$, obtêm-se alguns resultados diferentes. De fato, mais uma vez o intervalo de confiança no nível da mediana da regressão quantílica contém a estimativa por OLS; porém, para outros quantis, os resultados diferem (ver Apêndice 3): o impacto do coeficiente de abertura parece ser maior para países cuja taxa de crescimento da TFP é menor; ao passo que este impacto é significativamente menor a partir do quantil que deixa $70 \%$ à sua esquerda.

Outro teste de robustez consiste em abandonar a série de TFP e estimar o seguinte modelo alternativo, tendo como variável dependente a taxa média de crescimento da renda per capita:

$$
g_{y i}(0, t)=\beta_{0}+\beta_{1} \cdot \ln y_{i 0}+\beta_{2} \cdot \ln s_{k i}+\beta_{3} \cdot \ln s_{H i}+\beta_{4} \cdot \ln \left(n_{i}+g+\delta\right)+\beta_{5} A_{i}+\beta_{6} c p_{i}+\varepsilon_{i}
$$

onde a taxa de crescimento da renda per capita é calculada em log difference: $g_{y i}(0, t)=$ $\left(\ln y_{i t}-\ln y_{i 0}\right) /(t-0)$.

Em (15), as taxas de investimento em capital físico $\left(s_{k i}\right)$, em capital humano $\left(s_{H i}\right)$, e de crescimento populacional $\left(n_{i}\right)$, fundamentos do modelo de Solow, reaparecem como controles. Desse modo estamos isolando o impacto das nossas variáveis de interesse ( $c p$ e $A$ ) sobre a tecnologia. Colocando de outra maneira, a partir da forma estrutural do modelo de Solow com capital humano, Hall e Jones (1999) definem a produtividade total dos fatores como sendo equivalente ao erro de regressão 


$$
y_{y i}-\left[\beta_{0}+\beta_{1} \cdot \ln y_{i 0}+\beta_{2} \cdot \ln s_{k i}+\beta_{3} \cdot \ln s_{H i}+\beta_{4} \cdot \ln \left(n_{i}+g+\delta\right)\right]=T F P_{i}
$$

Os resultados da estimação de (15) encontram-se no Apêndice 4, e confirmam os resultados já obtidos: basicamente, a variável "composição" $(c p)$ aparece com sinal positivo e significância alta. As outras variáveis-fundamentos do modelo de Solow também aparecem com significância e os sinais esperados, inclusive $\beta_{1}<0$ (convergência).

\section{ENDOGENEIDADE}

Como se sabe, no primeiro estágio devemos regredir cada uma das variáveis supostamente endógenas, $c p$ (composição) e $A$ (abertura), contra as variáveis explicativas exógenas que estavam presentes nos modelos (12), (13) e (14), juntamente com um instrumento. Dessa forma, teremos as equações:

$$
\begin{gathered}
\left\{\begin{array}{l}
A_{i}=\beta_{0}+\beta_{1} . \text { instrumento }=\varepsilon_{i} \quad(17), \text { correspondendo a }(12) \\
c p_{i}=\beta_{0}+\beta_{1} . \text { instrumento }+\varepsilon_{i}
\end{array}\right. \\
\left\{\begin{array}{l}
A_{i}=\beta_{0}+\beta_{1} \cdot \ln T F P_{0 i}+\beta_{2} . \text { instrumento }+\varepsilon_{i} \quad(18), \text { correspondendo a }(13) \\
c p_{i}=\beta_{0}+\beta_{1} \cdot \ln T F P_{0 i}+\beta_{2} . \text { instrumento }+\varepsilon_{i}
\end{array}\right. \\
\left\{\begin{array}{l}
A_{i}=\beta_{0}+\beta_{1} \cdot \ln T F P_{0 i}+\beta_{2} \cdot \ln s_{H i}+\beta_{3} . \text { instrumento }+\varepsilon_{i} \quad(19), \text { correspondendo a }(14) \\
c p_{i}=\beta_{0}+\beta_{1} \cdot \ln T F P_{0 i}+\beta_{2} \cdot \ln s_{H i}+\beta_{3} . \text { instrumento }+\varepsilon_{i}
\end{array}\right.
\end{gathered}
$$

Conforme anunciado no final da Seção 2, testamos as variáveis que consideramos serem importantes instrumentos para explicar a Abertura Econômica. Na Tabela 4 podemos notar que para os modelos (17) e (18) todas as variáveis instrumentais se mostraram significativas, enquanto para o modelo (19) apenas a variável Law \& Order se mostrou estatisticamente igual a zero. Contudo, esses resultados favoráveis (significância de quase todos os instrumentos) da estimação pode se dever simplesmente a um baixo número de controles, especialmente nos modelos (17) e (18). A fim de corrigir esse efeito, testamos todos os instrumentos conjuntamente num mesmo modelo, como mostra a Tabela 5. Pelos resultados dessa tabela notamos que as variáveis que se mostram estatisticamente significantes são Investment Freedom e Strength of Investor's Protection.

Algo parecido acontece quando analisamos as regressões de primeiro estágio relativas à variável Composição dos fluxos de investimento: de fato, quando realizamos tais regressões com as variáveis instrumentais uma a uma (Tabela 6), a maioria se mostra estatisticamente significante; enquanto que ao estimarmos conjuntamente (Tabela 7) apenas a variável GP index se mostra relevante.

Agora que temos os instrumentos relevantes ao estudo (Investment Freedom e Strength of Investor's Protection para Abertura e GP Index para Composição), podemos substituí-los nos respectivos modelos para testar se as variáveis explicativas mantêm sua significância - o que nos leva ao segundo estágio da regressão:

Pelos resultados acima podemos notar que nos modelos (12') e (13'), correspondentes de 2 estágios a (12) e (13) da seção anterior, a variável referente à composição dos fluxos de investimentos se mostrou relevante a $10 \%$ e $5 \%$, respectivamente. A perda de significância no modelo (14') indica uma alta correlação entre o instrumento GP Index (proteção a patentes) e o capital humano $(\mathrm{sH})$ dos países: a inclusão do capital humano torna redundante a variável composição (estimada por GP Index no primeiro estágio). 
Tabelas 4 e 5: OLS - Regressão de Primeiro Estágio, tendo como variável dependente a Abertura Econômica dos países, estimada com variáveis instrumentais de forma individual e de forma conjunta, respectivamente

\begin{tabular}{lccc}
\hline \multicolumn{4}{c}{ Primeiro estágio: Testando instrumentos para Abertura, individualmente } \\
\hline \multicolumn{1}{c}{ Especificação } & Modelo (17) & Modelo (18) & Modelo (19) \\
\hline \multirow{2}{*}{ Inv Freedom } & $0.000641^{* *}$ & $0.000697^{*}$ & $0.000638^{* *}$ \\
& $(0.000245)$ & $(0.000256)$ & $(0.000277)$ \\
Property Rights & $0.000394^{* *}$ & $0.000454^{*}$ & $0.000422^{* * *}$ \\
& $(0.000164)$ & $(0.000165)$ & $(0.00022)$ \\
Law and Order Index & $0.004083^{* * *}$ & $0.004357^{* *}$ & 0.002327 \\
\multirow{2}{*}{ Strength of Inv's Protect } & $(0.002355)$ & $(0.002143)$ & $(0.002484)$ \\
& $0.008021^{* *}$ & $0.008183^{* *}$ & $0.007608^{* * *}$ \\
& $(0.003351)$ & $(0.003359)$ & $(0.003855)$
\end{tabular}

Primeiro estágio: Testando instrumentos para Abertura, conjuntamente

\begin{tabular}{lccc}
\hline Especificação & Modelo (17) & Modelo (18) & Modelo (19) \\
\hline \multirow{2}{*}{ Inv Freedom } & $0.000487^{* *}$ & $0.000496^{* *}$ & $0.000495^{* *}$ \\
& $(0.000229)$ & $(0.00024)$ & $(0.000239)$ \\
Property Rights & 0.0000449 & 0.000117 & 0.000125 \\
& $(0.000195)$ & $(0.000208)$ & $(0.000125)$ \\
Law and Order Index & -0.002594 & -0.002938 & -0.002919 \\
& $(0.002893)$ & $(0.002842)$ & $(0.002882$ \\
Strength of Inv's Protect & $0.006467^{* *}$ & $0.006289^{* *}$ & $0.006329^{* *}$ \\
& $(0.003044)$ & $(0.003057)$ & $(0.003318)$ \\
& & & \\
\hline
\end{tabular}

Nota: erros-padrão em parênteses.

*, ** e ${ }^{* * *}$ para significante a $1 \%, 5 \%$ e $10 \%$ respectivamente.

Fontes: Heritage.org, PRS Group, Doing Business. 
Tabelas 6 e 7: Regressão de Primeiro Estágio, tendo como variável dependente a Composição dos fluxos de investimentos dos países, estimadas de forma individual e de forma conjunta, respectivamente

\begin{tabular}{lccc}
\hline \multicolumn{3}{c}{ Primeiro estágio: Testando instrumentos para Composição, individualmente } \\
\hline \multicolumn{1}{c}{ Especificação } & Modelo (17) & Modelo (18) & Modelo (19) \\
\hline \multirow{2}{*}{ GP index } & $0.151530^{*}$ & $0.152271^{*}$ & $0.107632^{* *}$ \\
& $(0.042913)$ & $(0.049915)$ & $(0.052313)$ \\
Cost to export & $-0.000125^{*}$ & $-0.000125^{* *}$ & -0.0000395 \\
& $(0.0000469)$ & $(0.0000477)$ & $(0.000049)$ \\
Cost to enforce a contract & $-0.002342^{* *}$ & $-0.002157^{* *}$ & -0.0002 \\
Strength of Inv's Protect & $(0.000919)$ & $(0.000985)$ & $(0.000659)$ \\
& $0.038856^{* * *}$ & 0.035421 & 0.010632 \\
Investment Profile & $(0.022265)$ & $(0.021352)$ & $(0.027162)$ \\
& 0.011499 & 0.005972 & -0.016516 \\
& $(0.022658)$ & $(0.022887)$ & $(0.027877)$
\end{tabular}

\begin{tabular}{lccc}
\hline \multicolumn{3}{c}{ Primeiro estágio: Testando instrumentos para Composição, conjuntamente } \\
\hline Especificação & Modelo (17) & Modelo (18) & Modelo (19) \\
\hline \multirow{2}{*}{ GP index } & $0.174535^{* *}$ & $0.169002^{* *}$ & $0.146157^{* * *}$ \\
& $(0.071364)$ & $(0.078356)$ & $(0.077033)$ \\
Cost to export & -0.0000648 & 0.0000695 & -0.000051 \\
& $(0.0000536)$ & $(0.0000526)$ & $(0.0000488)$ \\
Cost to enforce a contract & -0.000157 & -0.000187 & 0.000313 \\
& $(0.000802)$ & 0.00083 & $(0.000819)$ \\
Strength of Inv's Protect & 0.029239 & 0.028598 & 0.023680 \\
& $(0.030852)$ & $(0.03131)$ & 0.033485 \\
Investment Profile & -0.035570 & -0.035763 & -0.036794 \\
& $(0.036164)$ & $(0.036158)$ & $(0.036210)$ \\
\hline
\end{tabular}

Nota: erros-padrão em parênteses.

*, ** $\mathrm{e}^{* * *}$ para significante a $1 \%, 5 \%$ e $10 \%$ respectivamente.

Fontes: Doing Business, Ginarte e Park (1997) e Park (2008), ICRG Risk Rating System. 
Tabela 8: 2SLS - Regressão de Segundo Estágio tendo como variável dependente a taxa média de crescimento da TFP em log diferença

\begin{tabular}{|c|c|c|c|}
\hline \multicolumn{4}{|c|}{$\begin{array}{l}\text { Segundo estágio: crescimento da TFP (1980 a 2006), } \\
\text { com os instrumentos significativos do primeiro estágio: } \\
\text { Investment Freedom, Strength of Investor's Protection e GP Index }\end{array}$} \\
\hline Especificação & Modelo (12') & Modelo (13’) & Modelo (14') \\
\hline \multirow{2}{*}{ Composição } & $0.026564^{* * *}$ & $0.046364^{* *}$ & 0.056484 \\
\hline & $(0.014174)$ & $(0.020597)$ & $(0.035709)$ \\
\hline \multirow{2}{*}{ Abertura } & 0.145139 & 0.097394 & 0.103537 \\
\hline & $(0.106258)$ & $(0.12823)$ & $(0.145849)$ \\
\hline \multirow{2}{*}{ ln TFP inicial } & & $-0.013627^{* *}$ & $-0.013968^{* * *}$ \\
\hline & & $(0.006162)$ & $(0.007151)$ \\
\hline \multirow{2}{*}{$\ln \mathrm{Sh}$} & & & -0.003282 \\
\hline & & & $(0.005394)$ \\
\hline \multirow{2}{*}{ Constante } & $-0.008803^{*}$ & $0.051484^{* * *}$ & 0.043391 \\
\hline & $(0.003045)$ & $(0.028619)$ & $(0.036952)$ \\
\hline \multicolumn{4}{|c|}{ Nota: erros-padrão em parênteses. } \\
\hline \multicolumn{4}{|c|}{$*{ }^{* *} \mathrm{e}^{* * *}$ para significante a $1 \%, 5 \%$ e $10 \%$ respectivamente. } \\
\hline \multicolumn{4}{|c|}{ Fontes: Untad, Barro e Lee (2001), PWT 7.0, Heritage.org, Doing Business e } \\
\hline \multicolumn{4}{|c|}{ Ginarte e Park (1997) e Park (2008). } \\
\hline
\end{tabular}

A constante também se mostrou significante nos dois primeiros modelos da Tabela 8 , sendo negativa no modelo (12') e positiva no modelo (13’); ou seja, após a inclusão do nível inicial da TFP dos países, o parâmetro relativo à constante ficou com sinal oposto, indicando que no modelo (12') esse parâmetro provavelmente estaria capturando parte da variabilidade relativa ao nível inicial da TFP; dessa forma, se mostrou válida a adição da variável ln TFP inicial nos modelos posteriores. Ademais, o fato de o parâmetro relativo ao nível inicial da TFP ter se mostrado negativo e estatisticamente significante nos modelos acima também condiz com a hipótese de convergência.

Comparando as Tabelas 3 e 8 , fica evidente por todas as especificações que a variável Abertura (FDI/PIB, grosso modo) guarda uma relação de determinação simultânea com o crescimento da TFP. Esse resultado está em linha com Mendonça e Nonnenberg (2005) que, analisando um painel de 33 países entre 1975 e 2000, concluíram que o FDI não causa, no sentido de Granger, o PIB. Apesar de o parâmetro referente à abertura econômica não ter se mostrado significante, é interessante atentarmos novamente ao impacto relativo das variáveis abertura e composição sobre a variável dependente. Ao invés de o impacto da abertura ser quase 17 vezes maior quando comparado ao da composição, como havia sido na regressão (14) da Tabela 3, ao incluirmos as variáveis instrumentais percebemos que esse impacto diminui para menos de 2 vezes em (14'), indicando novamente um possível efeito corretivo das variáveis instrumentais. 


\section{CONCLUSÃO}

No presente trabalho, testamos o impacto de 2 dois mecanismos de difusão internacional da tecnologia (FDI e licensing) sobre a taxa de crescimento da TFP. Para isso, propusemos especificações do modelo econométrico análogas à que Aghion et alii (2005) usaram para testar o impacto do desenvolvimento financeiro. Quanto a isso, se comparado ao trabalho de Pessoa (2008), o nosso tem como elemento original a inclusão da variável "composição" (royalties / FDI flow), que mede para cada país a importância relativa dos mecanismos licensing e FDI.

Num primeiro momento, ambas as variáveis explicativas dos nossos modelos, "abertura” ((FDI + royalties)/PIB) e "composição", se mostraram significativas para explicar o crescimento da TFP dos países da amostra, ambas aparecendo com os sinais positivos esperados. Além disso, as especificações que nós adotamos passaram todas elas nos testes de robustez realizados.

Num segundo momento, ao tratarmos do problema da endogeneidade, o resultado foi que encontramos instrumentos fortes no $1^{\circ}$. estágio, tanto para explicar "abertura”, quanto para explicar "composição". Além disso, o que é desejável do ponto de vista da intuição econômica, os instrumentos que explicam "abertura" (grosso modo, FDI) são variáveis institucionais diferentes daquelas que explicam "composição" (grosso modo, licensing). Contudo, no $2^{\circ}$. estágio, constatamos que no caso da variável "abertura", estamos diante de uma relação endógena ou de determinação simultânea com o crescimento da TFP.

Apenas a variável "composição" manteve sua significância no $2^{\circ}$. estágio, um resultado que nos é muito favorável quando nos lembramos de que o propósito principal deste trabalho era testar a conjectura, levantada a partir de Pessoa (2008), de que a transferência de tecnologia via licensing é mais favorável do que a via FDI ao crescimento da TFP de um país receptor. Pela nossa análise de 2 estágios, podemos dizer então que enquanto o FDI caminha pari passu com o crescimento econômico (da TFP), por outro lado existem variáveis institucionais exógenas ao crescimento e que o influenciam via licensing.

\section{BIBLIOGRAFIA}

Aghion, P., Howitt, P., \& Mayer-Foulkes, D. (2005). The effect of financial development on convergence: Theory and evidence. Quarterly Journal of Economics, 120:173-222.

Antràs, P. (2005). Incomplete contracts and the product cycle. American Economic Review, 95:1054-1073.

Barro, R. \& Lee, J. W. (2001). Schooling quality in a cross-section of countries. Economica, 68:465-489.

Bills, M. \& Klenow, P. (2000). Does schooling cause growth? American Economic Review, 90:1160-1183.

Cyhn, J. W. (2002). Technology Transfer and International Production: The development of the electronics industry in Korea. Edward Elgar, Cheltenham.

Ferreira, P., Galvão, A., gomes, F., \& Pessoa, S. (2010). The effects of external and internal strikes on total factor productivity. The Quarterly Review of Economics and Finance, 50:298-309.

Ginarte, J. C. \& Park, W. G. (1997). Determinants of patent rights: A cross-national study. Research Policy, 26:283-301.

Glass, A. J. \& Saggi, K. (2002). Licensing versus direct investment: Implications for economic growth. Journal of International Economics, 56:131-153.

Grossman, G. M. \& Helpman, E. (1991). Innovation and Growth in the Global Economy. MIT Press.

Hallock, K. F. \& Koenker, R. (2001). Quantile regression. Journal of Economic Perspectives, 15:143-56. 
Mankiw, N. g., romer, D., \& Weil, D. (1992). A contribution to the empirics of economic growth. Quarterly Journal of Economics, 107:407-438.

Mendonça, M. J. c. \& Nonnenberg, M. J. B. (2005). Determinantes dos investimentos diretos externos em países em desenvolvimento. Estudos Econômicos, 35.

Miller, S. M. \& Upadhyay, M. P. (2002). Total factor productivity and the convergence hypothesis. Journal of Macroeconomics, 24:267-287.

Park, W. G. (2008). International patent protection: 1960-2005. Research Policy Science Direct, 37:761-766.

Pessoa, S. (2008). Multinational corporations, foreign investment, and royalties and license fees: Effects on host-country total factor productivity. Notas Econômicas, 28:6-31.

Woo, J. (2009). Productivity growth and technological diffusion through foreign direct investment. Economic Inquiry, 47:226-248.

Zhao, M. (2006). Conducting R\&D in countries with weak intellectual property rights protection. Management Science, 52:1185-1199. 


\section{A. APÊNDICE 1: DESCRIÇÃO DAS VARIÁVEIS INSTITUCIONAIS USADAS COMO INSTRUMENTOS}

1) Investment Freedom Index - realizado pelo Index of Economic Freedom. Tal índice leva em conta as restrições que cada país adota em relação ao fluxo de capital para investimentos. Há diversas formas de restrição, por exemplo: alguns países impõem restrições a pagamentos e transferências de capital, algumas indústrias podem ser fechadas ao investimento estrangeiro e, ainda, alguns países podem restringir o acesso ao mercado de câmbio. Este índice varia entre 0 e 100, e valores mais próximos a 100 indicam um país com menos restrições.

2) Property Rights Index - realizado pelo Index of Economic Freedom. Este índice tenta capturar a habilidade dos indivíduos de um país em acumular propriedade privada, assegurada por leis claras que são reforçadas pelo Estado. Ele também leva em conta a probabilidade de expropriação da propriedade privada e a independência e a existência de corrupção no judiciário. Este índice varia entre 0 e 100, valores mais altos indicam um melhor sistema judicial e de aplicação de leis, assim como uma maior segurança para o investidor privado.

3) Law and Order Index - realizado pelo ICRG Risk Rating System. Este índice tenta capturar a força e imparcialidade do sistema legal do país juntamente com a visão que a população possui acerca da sua própria lei. Este índice varia entre 0 e 6 , e valores mais altos indicam uma maior força e imparcialidade do sistema legal.

4) Investment Profile Index - realizado pelo ICRG Risk Rating System. Este índice tenta capturar os riscos de um investimento que não são cobertos por outros riscos políticos, econômicos e/ou financeiros. Os principais fatores de risco analisados por tal índice são: viabilidade dos contratos/expropriação, repatriação de lucros e atraso de pagamentos. Este índice varia entre 0 e 12, e valores mais altos indicam um risco mais baixo.

5) Strength of Investor's Protection - realizado pelo relatório Doing Business do Banco Mundial. Este índice mede a força de proteção dos acionistas minoritários dentro das companhias. Apesar de estar um pouco fora do escopo deste estudo, decidimos testá-lo nas regressões já que consideramos que ele pode ser uma boa proxy tanto para o nível de desenvolvimento dos mercados financeiros dos países como do sistema legal dos mesmos. Este índice varia de 0 a 10 e valores mais altos indicam uma melhor proteção ao investidor.

6) Cost to enforce a contract - realizado pelo relatório Doing Business do Banco Mundial. Ele mede o custo, em percentuais de renda per capita, com todo o processo de defesa de contratos em corte. Ele é um indicador do quão eficiente são as medidas de proteção dos contratos, no sistema judiciário.

7) Cost to export - realizado pelo relatório Doing Business do Banco Mundial. Este índice compila o custo médio em dólares americanos dos requerimentos processuais para a exportação de uma carga padronizada de bens por via marítima. Consideramos que este índice poderia ser uma boa proxy para a decisão das empresas entre optar pelo licensing ou FDI. Uma vez que o licensing normalmente necessita mais do canal de exportação se comparado ao FDI; esperamos que quanto maior o custo de exportação, maior seria o incentivo a realização do FDI.

8) GP Index - conforme explicado acima, este índice realizado inicialmente em 1997 por Ginarte \& Park e, posteriormente, atualizado por Park em 2008, mede os direitos relacionados à proteção de patentes e dá uma nota que varia entre 0 e 5 , sendo notas mais altas para uma maior proteção. 


\section{B. APÊNDICE 2 : EVOLUÇÃO DO COEFICIENTE DA VARIÁVEL DE COMPOSIÇÃO DE FLUXOS ESTRANGEIROS EM FUNÇÃO DOS QUANTIS}

- Comparação de resultados entre quantílica e OLS para o modelo TFP com capital humano, especificação (14)

$\left(c p_{i} \equiv\left(\frac{\text { royalties }}{F D I}\right)_{i}\right)$

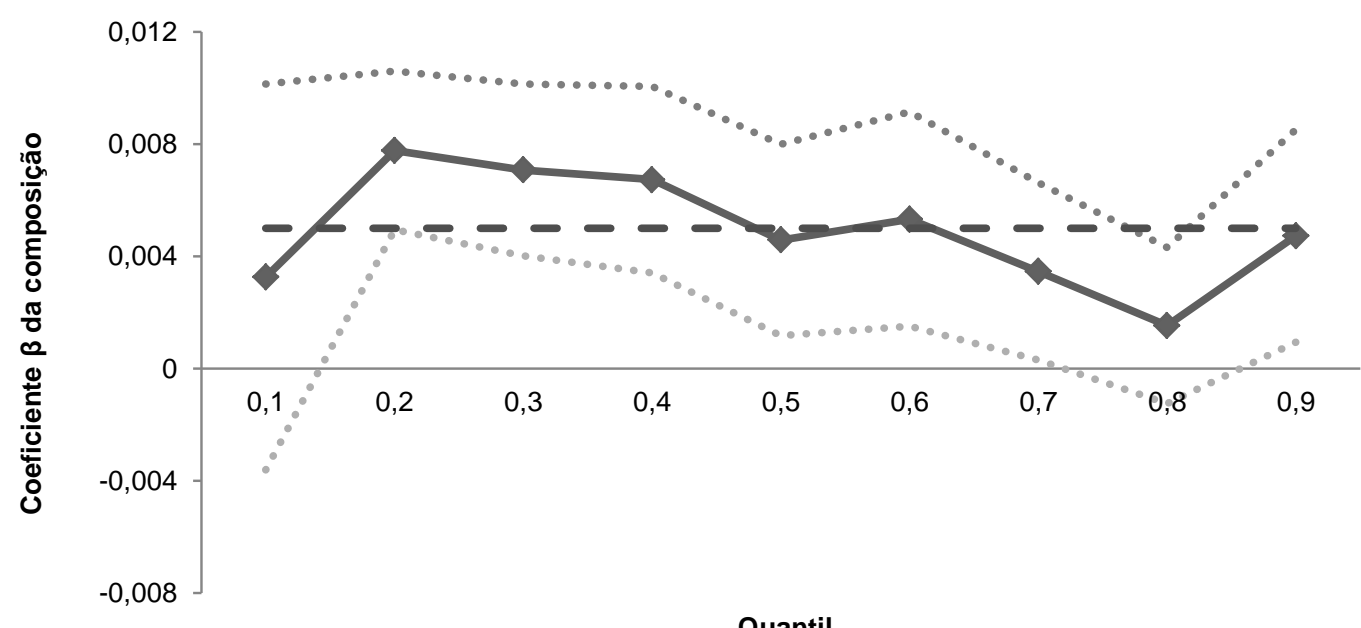

\section{Quantil}

$\longrightarrow$ Quantilica $\quad \ldots$. Intervalo de confiança $\quad-\quad$ OLS

Fonte: UNCTAD, Barro e Lee (2001) e Penn World Table 7.0. 


\section{APÊNDICE 3: EVOLUÇÃO DO COEFICIENTE DA VARIÁVEL DE ABERTURA A FLUXOS ESTRANGEIROS EM FUNÇÃO DOS QUANTIS}

- Comparação de resultados entre quantílica e OLS para o modelo TFP com capital humano, especificação (14)

$\left(A_{i} \equiv\left(\frac{\text { royalties }+F D I}{P I B}\right)_{i}\right)$

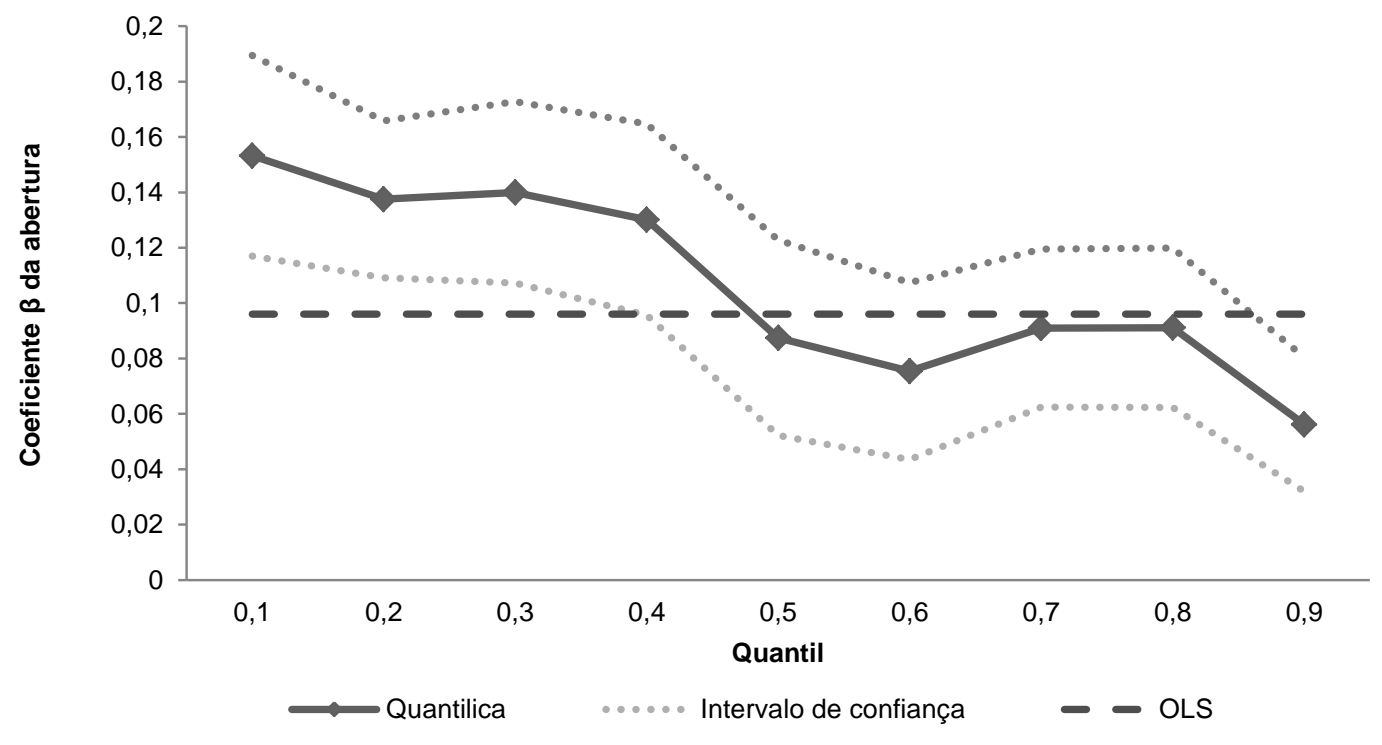

Fonte: UNCTAD, Barro e Lee (2001) e Penn World Table 7.0. 
D. APÊNDICE 4: REGRESSÃO HÍBRIDA (OLS) TENDO COMO VARIÁVEL DEPENDENTE $O$ CRESCIMENTO DO PIB PER CAPITA EM LOG DIFERENÇA

\begin{tabular}{cc}
\hline "Crescimento do PIB per capita \\
(log diferença) entre 1980 e 2006" \\
\hline ln sk & $0,008013^{* *}$ \\
$(0,0039)$ & $0,010016^{*}$ \\
ln sh & $(0,0038)$ \\
& $-0,035204$ \\
ln (n+g +delta) & $(0,0221)$ \\
& $-0,009025^{*}$ \\
ln yo & $(0,0026)$ \\
& $0,013290^{*}$ \\
Composição & $(0,0043)$ \\
& $0,084296^{* *}$ \\
Abertura & $(0,0405)$ \\
& 0,04554 \\
Constante & $(0,0559)$ \\
\hline Nota: erros-padrão em parênteses. \\
*,** e *** para significante a \\
1\%, 5\% e 10\% respectivamente. \\
Fontes: Untad, Barro e Lee $(2001)$ e \\
Penn World Table 7.0
\end{tabular}

